\title{
Dissociation of Subjective and Objective Alertness During Prolonged Wakefulness
}

\author{
Chao Hao' \\ Mingzhu $\mathrm{Li}^{1}$ \\ Wei Luo ${ }^{2}$ \\ Ning $M a \mathbb{D}^{\prime}$ \\ 'Key Laboratory of Brain, Cognition and \\ Education Sciences (South China Normal \\ University), Ministry of Education; \\ Center for Sleep Research, Center for \\ Studies of Psychological Application, \\ Guangdong Key Laboratory of Mental \\ Health and Cognitive Science, School of \\ Psychology, South China Normal \\ University, Guangzhou, 51063I, People's \\ Republic of China; ${ }^{2}$ School of \\ Architecture and Urban Planning, \\ Shenzhen University, Shenzhen, 518060, \\ People's Republic of China
}

Correspondence: Ning Ma School of Psychology, South China Normal University, Guangzhou, 5I063I, People's Republic of China

Email maning@m.scnu.edu.cn
Background: Although the deterioration of subjective and objective alertness during prolonged wakefulness has been investigated rigorously, whether perceived sleepiness and fatigue are consistent with actual decrements in behavioral performance in the time course is still disputed. The present study examined the dissociation between decrements of subjective alertness and performance deficits during prolonged wakefulness of one night and explored the relationship between body temperature and the impairments of subjective and objective alertness.

Participants and Methods: Thirty-eight participants (27 females; age: $21.76 \pm 2.37$ years old) underwent prolonged wakefulness for one night at habitual bedtime (0:00-6:00 am). Participants completed a 10-min PVT to assess objective alertness, fatigue, and sleepiness ratings to assess subjective alertness every 2 hours, and body temperature was measured every hour during scheduled wakefulness.

Results: Subjective alertness reflected a linear decline with time, but the magnitudes of objective performance deterioration increased significantly between 4:00 and 6:00 am. The increasing magnitudes of performance deficits were associated with the change of body temperature between 4:00 and 6:00 am.

Conclusion: These results indicate that the perceived degree of decline in alertness is temporally dissociated with the actual decline in objective vigilance with increased duration of wakefulness. The dissociation of magnitudes of subjective and objective alertness decrements mainly occurs between 4:00 and 6:00 am, and the changes of performance deficits have a relationship with body temperature.

Keywords: prolonged wakefulness, subjective ratings, objective performance, dissociation, body temperature

\section{Introduction}

It is increasingly common that prolonged wakefulness for study, working, and even entertainment happens in modern society. These working and lifestyle demands frequently require individuals to sacrifice their regular sleep schedules, resulting in sleep loss and circadian misalignment. Prolonged wakefulness across the night is associated with increases in subjective fatigue and sleepiness and a broad range of decrements in objective performance, including longer response time and more lapses in cognitive performance, particularly for alertness and sustained attention. ${ }^{1,2}$ Measures of sleepiness and fatigue can be assessed using subjective ratings like the Stanford Sleepiness Scale (SSS) and Karolinska sleepiness scale (KSS). ${ }^{3-5}$ Various behavioral tests have been developed to objectively assess the degree of cognitive performance deterioration related to prolonged wakefulness. ${ }^{6,7}$ 
Multiple studies have analyzed the temporal variations of decrements of subjective evaluations and objective performance during prolonged wakefulness periods. ${ }^{8-11}$ Subjective ratings and objective performance deteriorate significantly when wakefulness is extended into the usual bedtime, and the most severe deterioration emerges in the early morning hours. ${ }^{10,11}$ Despite continued wakefulness, both subjective ratings and objective performance were improved after the early morning. Because the circadian process represents a nearly $24 \mathrm{~h}$ oscillation, promoting wakefulness across the habitual daytime. ${ }^{8,12}$ However, other studies have revealed that decrements of subjective ratings and objective performance are not quantitatively correlated. ${ }^{13-15}$ The actual decline in objective vigilance maybe not consistent with individuals' perceived degrees of fatigue and sleepiness during sleep loss.

Subjective and objective alertness involve different brain oscillations. Decrements in subjective ratings are related to a decreased EEG activity in the high alpha range. However, the impairments of objective performance are related to an increase in EEG delta activity and reaction time is also highly correlated to EEG gamma. ${ }^{14,16,17}$ Although the relationship between subjective and objective alertness has been examined, it is not clear whether prolonged wakefulness affected subjective alertness and objective performance to different extents.

Prolonged wakefulness across the night also influences the physiological statuses controlled by the peripheral nervous system, including body temperature, heart rate, and blood pressure. ${ }^{18}$ Sleep restriction potentially affects the circadian rhythms of body temperature, resulting in dysfunctional control of body temperature. ${ }^{19}$ Mean body temperature significantly decreases during the sleep restriction condition compared to the natural sleep condition. $^{20-22}$ When sleep deprivation lasts for more than one night, heart rate decreases due to increased vagal activity, whereas shorter periods of sleep deprivation do not seem to affect heart rate. ${ }^{22-24}$ Previous studies have reported that blood pressure is elevated following acute total sleep deprivation and partial sleep restriction. ${ }^{25,26}$ The peripheral nervous system is controlled by a neural system named the central autonomic network (CAN). Within the CAN, the integrated activity of respiratory, cardiovascular, and thermoregulatory regulation, together with behavioral responses, operates to maintain homeostasis of the human body. ${ }^{18,27}$ The hypothalamus modulates the timing of sleep and wakefulness, as well as waking behavior, such as sleepiness and cognitive performance. ${ }^{28}$
The physiological activity changes with wake and sleep states; in turn, the physiological activity also modulates the wake-sleep state. ${ }^{27}$ The alternations of physiological functions are associated with the changes in neurobehavioral performance. An increased body temperature is correlated with improved performance and alertness. ${ }^{29}$ Objective performance deterioration may be partly attributed to disruption of body temperature rhythms. ${ }^{30}$ Heart rate variability can be used to estimate sleepiness-related decrements in psychomotor vigilance during total sleep deprivation. ${ }^{13}$ The changes in physiological functions due to circadian misalignment may correlate with the deterioration in subjective ratings and objective performance during prolonged wakefulness periods.

The present study aimed to examine the effects of prolonged wakefulness overnight on the objective performance on PVT and subjective ratings of fatigue and sleepiness and whether the magnitudes of increase in subjective ratings temporally correspond with the decrease in objective performance. This research also analyzed the potential relationship of body temperature variations with the changes in subjective alertness decrements and performance deficits during prolonged wakefulness. We have hypothesized that both subjective and objective alertness deteriorated continuously with time on task during prolonged wakefulness periods. However, the rate of change in subjective alertness was temporally inconsistent with the rate of variation in performance impairments. Previous research has established a positive relationship between daily rhythms of body temperature and cognitive performance in humans. ${ }^{9,29-31}$ According to previous studies, we hypothesized that the changes in performance deficits were associated with the variation in body temperature during periods of prolonged wakefulness.

\section{Participants and Methods}

\section{Participants}

Forty-two healthy volunteers completed this study, which was conducted in the Center for Sleep Research at the South China Normal University. Four participants were excluded as outliers with an absolute value of standardized residuals greater than $3 .^{32}$ Therefore, the final sample consisted of thirty-eight participants $\left(\mathrm{M}_{\mathrm{age}}=21.76 \pm\right.$ 2.37 years old, 27 females) with normal body mass index $\left(20.86 \pm 1.75 \mathrm{~kg} / \mathrm{m}^{2}\right)$ and normal or corrected-to-normal vision. All study procedures were approved by the Ethical Committee of the South China Normal University and 
were conducted in accordance with the Declaration of Helsinki. All participants provided written informed consent and were financially compensated for their time.

Their demographic information, sleep and health history were assessed by a structural screening questionnaire. They were all nonsmokers and had no past or present major physical, psychiatric, and sleep-related disorders. Participants were ineligible if they had a history of shift work or traveling across time zones within two months before starting the study. Three days before the study, participants were asked to avoid alcohol, caffeine, or medications. Participants were also required to maintain a stable daily sleep-wake schedule (habitual 6.5-8 hours' sleep duration and awake times between 6:00 am and 9:00 am) within two weeks before the study, verified by sleep diaries and actigraphy (Actiwatch Spectrum, Philips).

\section{Materials and Methods}

\section{Experimental Procedures}

Participants attended the sleep laboratory at 19:00 and were kept awake at habitual bedtime (0:00-6:00 am) in the lab. This period was monitored by researchers to ensure participants' wakefulness. All the tests began at 0:00 am. Participants completed a 10-min psychomotor vigilance task $(\mathrm{PVT})^{6}$ to assess objective alertness and a series of subjective tests including fatigue and sleepiness ratings during scheduled wakefulness every 2 hours. Physiological tests, including body temperature, systolic pressure, diastolic pressure, and heart rate were measured every hour. All the subjective ratings and physiological measurements were conducted before the PVT, avoiding the interference from fatigue effects induced by PVT (Figure 1).

\section{Psychomotor Vigilance Test}

Psychomotor vigilance test (PVT) is sensitive to sleep loss, which is a simple reaction time task and requires individuals to respond to stimuli that occur at random intervals as quickly as possible. ${ }^{33,34}$ PVT is highly reliable and be virtually unaffected by learning. ${ }^{6}$ We applied the standard 10-min PVT protocol (adapted version of Dinges and Powell, 1985) with random visual stimuli varied randomly from $2-10$ s. $^{35}$ The number of trials varied from 70 to 90, depending on the participants' reaction speed. Participants were instructed to monitor a red rectangular box on the computer screen and press a response button as soon as a yellow stimulus appeared on the screen, after which they would be able to view their reaction time. Button presses when the red rectangular box is not displayed on the screen are counted as false starts or errors of commission, which participants are instructed to avoid (Figure 2).

The mean 1/RT and the number of lapses were chosen as the primary PVT indices, which maximize sleep loss sensitivity. ${ }^{6,36}$ Responses were assessed as valid if RT was $\geq 100 \mathrm{~ms}$. Lapses were counted when RT was $\geq 500 \mathrm{~ms}$. If there was a $30 \mathrm{~s}$ period without response, stopped timing and counted as a lapse with $30 \mathrm{~s}$ response time. For calculating mean 1/RT, each RT (ms) was divided by 1000 and then reciprocally transformed. 1/RT emphasizes slowing in the optimum and intermediate response domain, and it substantially decreases the contribution of long lapses. ${ }^{6}$

\section{Subjective and Physiological Test}

Fatigue was assessed by a 9-point Likert scale ranging from " $1 "=$ not at all fatigue to " $9 "=$ extremely fatigue. Participants rated their sleepiness by completing the Karolinska Sleepiness Scale (KSS). ${ }^{3}$ The KSS is a 1 -item visual analogue scale, scored using a 9-point response range, from 1 (extreme alertness), to 5 (neither alertness nor sleepiness), to 9 (extreme sleepiness). Body temperature was measured by an electronic oral thermometer (Omron Healthcare Co, Ltd., Kyoto, Japan). Systolic pressure, diastolic pressure, and heart rate were measured by an automatic sphygmomanometer (Omron Healthcare Co, Ltd., Kyoto, Japan), and the experimenter measured the blood pressure after the training.

\section{Statistical Analysis}

The normality of data was tested using the Shapiro-Wilk test. Comparisons of differences of subjective (fatigue

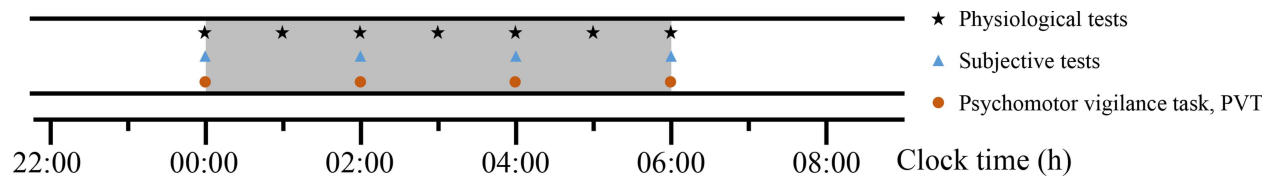

Figure I Protocol for PVT performance, physiological and subjective tests. During habitual bedtime, participants kept awake for 6 hours (shown in shadow). Participants completed a 10-min PVT (indicated by circles, ) and subjective ratings (indicated by triangles, $\Delta$ ) every two hours. The physiological tests were conducted within each hour (indicated by asterisks, $\underset{\wedge}{\wedge}$ ). 


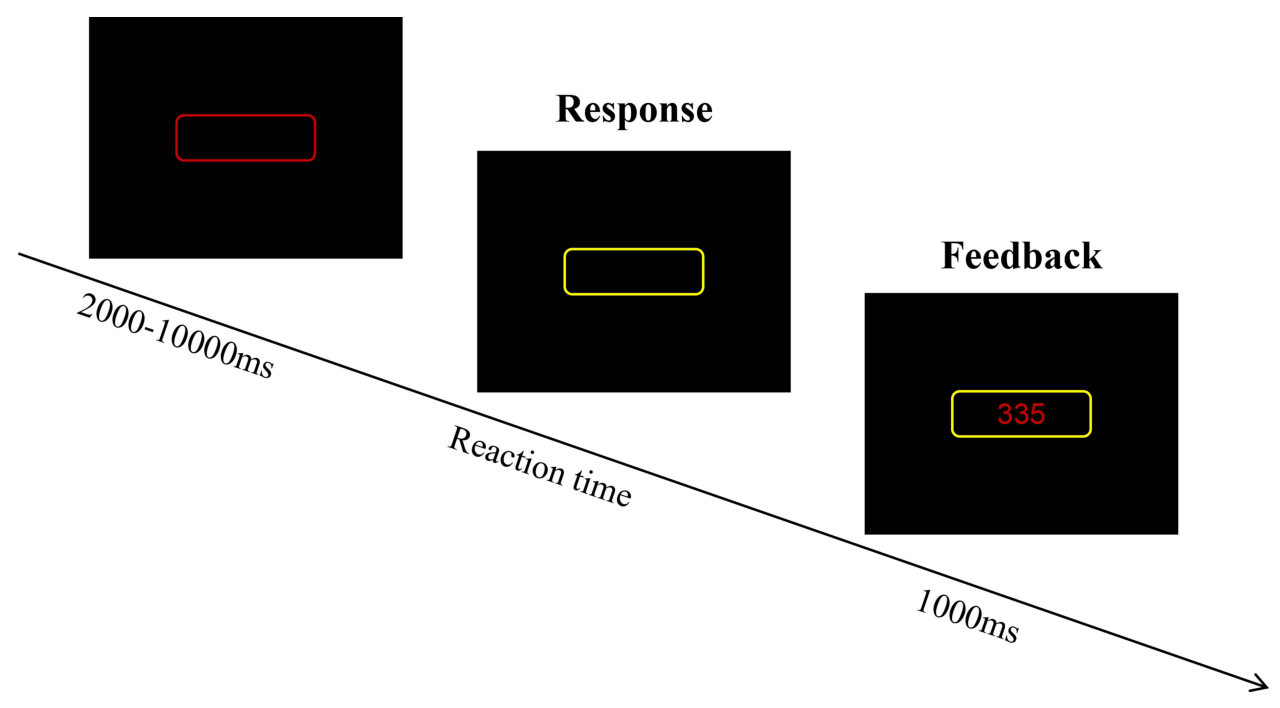

Figure 2 Psychomotor vigilance test (PVT) task.

and sleepiness), objective (number of lapses and mean 1/ RT), and physiological (temperature, systolic pressure, diastolic pressure, and heart rate) outcome metrics among prolonged wakefulness time (0:00, 2:00, 4:00, and 6:00 am) were carried out using Repeated Measurement ANOVA for normal-distributed data and Friedman tests for non-normal-distributed data. Then, to explore the variation trends of subjective ratings, objective performance, and physiological indexes, the present study calculated the relative changes in the adjacent time (2:00 minus 0:00, 4:00 minus 2:00, and 6:00 minus 0:00 am) in these variables. The magnitudes of the relative changes in the adjacent time marked as $\Delta$ subjective ( $\Delta$ fatigue and $\Delta$ sleepiness), $\Delta$ objective ( $\Delta$ number of lapses and $\Delta$ mean $1 / \mathrm{RT}$ ), and $\Delta$ physiological ( $\Delta$ temperature, $\Delta$ systolic pressure, $\Delta$ diastolic pressure, and $\Delta$ heart rate) outcome metrics, which were also contrasted by Repeated Measures ANOVA for normal-distributed data and Friedman tests for non-normal-distributed data. Finally, we conducted Pearson correlation analysis for normal-distributed data and Spearman correlation analysis for non-normal-distributed data among the $\Delta$ subjective, $\Delta$ objective, and $\Delta$ physiological outcome metrics to explore the relationship among the relative changes of subjective, objective, and physiological status across the period of prolonged wakefulness. We computed respectively the correlation for each point of time $(0: 00,2: 00$, 4:00, and 6:00 am). Analyses were performed using SPSS23.0. The statistical significance level was set at $p<0.05$ (two-tailed) for all tests. For post-hoc analysis, the Bonferroni test was applied for $p$-value adjustments of multiple comparisons, and corrected $p$-values are reported.

\section{Results}

\section{Assessment of the Absolute Changes}

The time courses of the subjective, objective, and physiological outcome metrics from 0:00 to 6:00 am are shown in Figure 3. Differences of objective, subjective indexes and body temperature among 0:00, 2:00, 4:00, and 6:00 were significant (Table 1). The Friedman tests revealed a significantly increasing number of lapses (Figure 3A; $\left.\chi_{(3)}^{2}=49.822, \quad p<0.001\right)$ and a significantly decreasing response speed (Figure $3 \mathrm{~B} ; \chi^{2}$ (3) $=72.474, p<0.001)$ over time. Post hoc tests showed that the number of lapses was more at 6:00 than 4:00 ( $p=$ 0.006 , Bonferroni corrected), as well as the mean response speed was slower at 6:00 than 4:00 $(p<0.001$, Bonferroni corrected). There was a significant increase in sleepiness (Figure 3C; $\chi_{(3)}^{2}=79.917, p<0.001$ ) and fatigue (Figure $3 \mathrm{D} ; \chi_{(3)}^{2}=74.146, p<0.001$ ) from midnight to early morning. Post hoc tests showed that participants were sleepier and more fatigue at 2:00 than 0:00 (sleepiness, $p=0.023$, Bonferroni corrected; fatigue, $p=$ 0.040, Bonferroni corrected), as well as at 6:00 than 4:00 (sleepiness, $p=0.013$, Bonferroni corrected; fatigue, $p=$ 0.010, Bonferroni corrected). A similar reduction of result was observed for body temperature (Figure 3E; $F$ $(3,111)=12.559, p<0.001)$. Post hoc tests showed that body temperature was lower at 2:00 than 0:00 $(p<0.001$, 

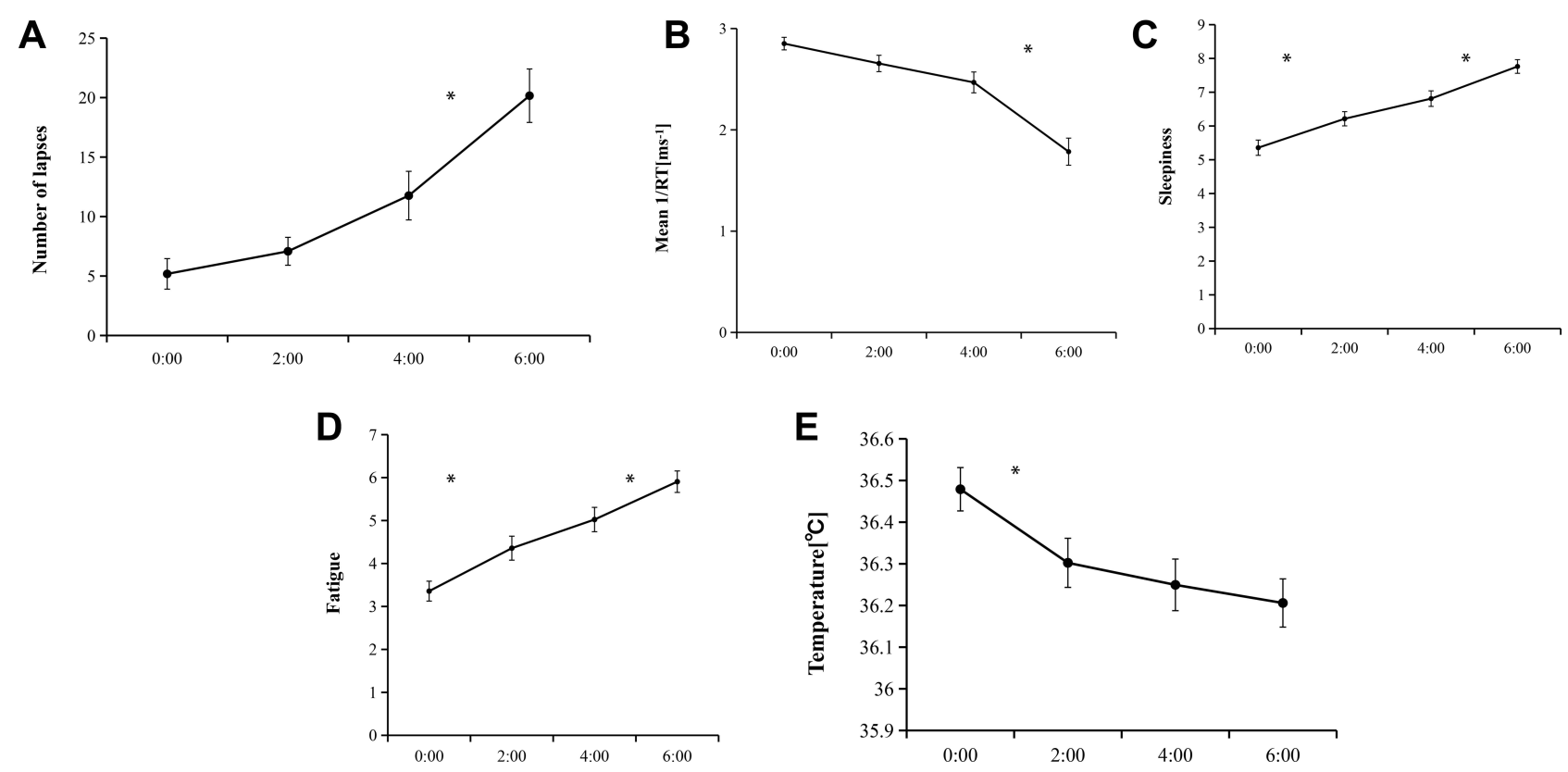

Figure 3 The time courses of the subjective, objective, and physiological outcome metrics from 0:00 to 6:00 am. (A) Number of lapses, (B) Mean I/RT, (C) Sleepiness, (D) Fatigue, (E) Body temperature. Asterisks indicate significant differences, ${ }^{*} p<0.05$. In each plot, the mean \pm SEM is shown.

Bonferroni corrected). There was no significant difference in systolic pressure, diastolic pressure, and heart rate among 0:00, 2:00, 4:00 and 6:00 am (Table S1, systolic pressure, $p=0.217$; diastolic pressure, $p=$ 0.053; heart rate, $p=0.605$ ).

\section{Assessment of the Relative Changes in Adjacent Time}

To assess all outcome metrics' variation trends, we conducted the Friedman tests on $\triangle \mathrm{PVT}, \Delta$ subjective, and $\Delta$ physiological indices (Table 2). There were significant

Table I Results of Absolute Values of Objective, Subjective, and Physiological Outcome Metrics Over the Course of Time (0:00, 2:00, 4:00, and 6:00 Am)

\begin{tabular}{|c|c|c|c|c|c|c|c|}
\hline \multicolumn{2}{|c|}{ Outcome Metrics } & \multirow{2}{*}{$\begin{array}{l}0: 00 \\
5.18 \pm 7.91 \\
2.90 \pm 0.35\end{array}$} & \multirow{2}{*}{$\begin{array}{l}2: 00 \\
7.08 \pm 7.24 \\
2.74 \pm 0.43\end{array}$} & \multirow{2}{*}{$\begin{array}{l}4: 00 \\
11.76 \pm 12.59 \\
2.56 \pm 0.58\end{array}$} & \multirow{2}{*}{$\begin{array}{l}6: 00 \\
20.16 \pm 13.85 \\
1.84 \pm 0.80\end{array}$} & \multirow{2}{*}{$\begin{array}{l}\chi^{2} / \mathbf{F} \\
49.822^{* * *} \\
72.474^{* * * *}\end{array}$} & \multirow{2}{*}{$\begin{array}{l}\mathbf{P} \\
<0.001 \\
<0.001\end{array}$} \\
\hline PVT & $\begin{array}{l}\text { Number of lapses } \\
\text { Mean I/RT }\left(\mathrm{ms}^{-1}\right)\end{array}$ & & & & & & \\
\hline Subjective & $\begin{array}{l}\text { Sleepiness } \\
\text { Fatigue }\end{array}$ & $\begin{array}{l}5.71 \pm 1.16 \\
3.71 \pm 1.20\end{array}$ & $\begin{array}{l}6.53 \pm 1.29 \\
4.55 \pm 1.48\end{array}$ & $\begin{array}{l}7.03 \pm 1.30 \\
5.16 \pm 1.55\end{array}$ & $\begin{array}{l}8.08 \pm 1.02 \\
6.24 \pm 1.73\end{array}$ & $\begin{array}{l}79.917 * * * \\
74.146 * * *\end{array}$ & $\begin{array}{l}<0.001 \\
<0.001\end{array}$ \\
\hline Physiological & Temperature $\left({ }^{\circ} \mathrm{C}\right)$ & $36.48 \pm 0.33$ & $36.32 \pm 0.34$ & $36.29 \pm 0.38$ & $36.23 \pm 0.35$ & $12.559 * * *$ & $<0.001$ \\
\hline
\end{tabular}

Notes: $* * * p<0.001$. Values are shown as mean \pm SD. The test statistic of number of lapses, mean I/RT, sleepiness, and fatigue is $\chi^{2}$, and the test statistic of temperature is $F$.

Table 2 Results of Relative Values of $\Delta$ objective, $\Delta$ subjective, and $\Delta$ physiological Outcome Metrics Over Time

\begin{tabular}{|c|c|c|c|c|c|c|}
\hline \multicolumn{2}{|c|}{ Outcome Metric } & $2: 00-0: 00$ & $4: 00-2: 00$ & $6: 00-4: 00$ & $\chi^{2}$ & $\mathbf{P}$ \\
\hline PVT & $\begin{array}{l}\Delta \text { Number of lapses } \\
\Delta \text { Mean I/RT }\left(\mathrm{ms}^{-1}\right)\end{array}$ & $\begin{array}{l}1.89 \pm 6.27 \\
-0.16 \pm 0.25\end{array}$ & $\begin{array}{l}4.68 \pm 9.53 \\
-0.18 \pm 0.33\end{array}$ & $\begin{array}{l}8.39 \pm I I .76 \\
-0.7 I \pm 0.70\end{array}$ & $\begin{array}{l}11.65 I^{* * *} \\
23.21 I^{* * *}\end{array}$ & $\begin{array}{l}0.003 \\
<0.001\end{array}$ \\
\hline Subjective & $\begin{array}{l}\Delta \text { Sleepiness } \\
\Delta \text { Fatigue }\end{array}$ & $\begin{array}{l}0.82 \pm 0.95 \\
0.84 \pm 0.89\end{array}$ & $\begin{array}{l}0.50 \pm 0.89 \\
0.61 \pm 1.10\end{array}$ & $\begin{array}{l}1.05 \pm 1.14 \\
1.08 \pm 1.15\end{array}$ & $\begin{array}{l}4.049 \\
3.907\end{array}$ & $\begin{array}{l}0.132 \\
0.142\end{array}$ \\
\hline Physiological & $\Delta$ Temperature $\left({ }^{\circ} \mathrm{C}\right)$ & $-0.16 \pm 0.21$ & $-0.04 \pm 0.25$ & $-0.06 \pm 0.25$ & 2.080 & 0.353 \\
\hline
\end{tabular}

Notes: $*_{p}^{*}<0.0 \mathrm{I}$, and $*_{* *}<0.00 \mathrm{I}$. The magnitudes of the relative changes in the adjacent time marked as $\Delta$ subjective ( $\Delta$ fatigue and $\Delta$ sleepiness), $\Delta$ objective $(\Delta$ number of lapses and $\Delta$ mean I/RT), and $\Delta$ physiological ( $\Delta$ temperature) outcome metrics. Values are shown as mean \pm SD. 
differences in the magnitudes of objective performance deterioration in the adjacent time ( $\Delta$ number of lapses, $\chi_{(2)}^{2}=$ 11.651, $p=0.003$; and $\Delta$ mean $1 / \mathrm{RT}, \chi_{(2)}^{2}=23.211, p<$ 0.001). Post hoc tests found a significant difference of $\Delta$ mean 1/RT between 4:00 minus 2:00 and 6:00 minus 4:00 ( $p<0.001$, Bonferroni corrected). However, the extents of increases in sleepiness and fatigue were steady all the time ( $\Delta$ sleepiness, $\chi_{(2)}^{2}=4.049, p=0.132 ; \Delta$ fatigue, $\chi_{(2)}^{2}=3.907$, $p=0.142$ ). There was no significant difference in $\Delta$ body temperature, $\Delta$ systolic pressure, $\Delta$ diastolic pressure, and $\Delta$ heart rate among 0:00, 2:00, 4:00 and 6:00 am ( $\Delta$ body temperature, $p=0.353$; Table $\mathrm{S} 2, \Delta$ systolic pressure, $p=$ 0.241; $\Delta$ diastolic pressure, $p=0.529 ; \Delta$ heart rate, $p=0.418$ ).

\section{Correlation Analysis Among the Relative Changes in the Adjacent Time}

To explore the relationship between the changes in physiological indices and the changes in objective and subjective alertness, we conducted Pearson or Spearman correlation analysis among $\Delta$ subjective, $\Delta$ objective indexes and $\Delta$ physiological indices (Table 3). Between 0:00 and 2:00 and between 2:00 and 4:00, no $\Delta$ objective or $\Delta$ subjective outcome metrics were correlated to $\Delta$ body temperature. From 4:00 to 6:00, $\Delta$ temperature was significantly associated with $\Delta$ number of lapses (Figure $4 \mathrm{~A} ; r=$ $-0.374, p=0.021$ ) and $\Delta$ mean $1 / \mathrm{RT}$ (Figure 4B; $r=0.372$, $p=0.021$ ). The magnitudes of objective alertness deterioration were correlated to the changes in body temperature between 4:00 and 6:00. At all the time points, $\Delta$ systolic pressure, $\Delta$ diastolic pressure, and $\Delta$ heart rate were not correlated to the $\Delta$ objective and $\Delta$ subjective outcome metrics (Table S3).

\section{Discussion}

The present study explored the absolute and relative changes of subjective ratings and objective performance respectively and analyzed the relationship between the variations of body temperature and the impairments of subjective and objective alertness during the period of prolonged wakefulness. This study found the expected dissociation between the magnitudes of the impairments of subjective and objective alertness temporally. The rate of PVT performance decrements was significantly increased between 4:00 and 6:00. However, the changes of sleepiness and fatigue were stable all the time. The increasing rate of objective alertness decrements was associated with the change of body temperature between 4:00 and 6:00.

Both subjective ratings and objective performance kept decline during the period of prolonged wakefulness, and the most severe deterioration occurred between 4:00 and 6:00 am. The temporal variations of both subjective and objective alertness during prolonged wakefulness are under the dual control of a homeostatic process and a circadian rhythm. The homeostatic drive for sleep competes to remain awake, resulting in a rise in sleep pressure with increasing awake time. The circadian process represents a nearly $24 \mathrm{~h}$ oscillation, promoting sleepiness across the habitual bedtime $(0: 00-6: 00 \mathrm{am}){ }^{8,12,37}$ During extended wakefulness across the night, the interaction of the dual processes leads to time-on-task decrements in subjective ratings and objective performance.

Although subjective alertness has a similar temporal pattern of alterations to objective alertness, perceived sleepiness and fatigue are inconsistent with actual decrements in behavior performance when wakefulness is extended into the biological night. Reaction speed in a vigilance task suffered significant decrements between 4:00 and 6:00, but participants had not perceived the increasing magnitudes of the objective alertness deterioration. It indicates that significant decrements of objective performance on vigilance tasks do not temporally correspond with high levels of sleepiness and fatigue between 4:00 and 6:00. Individuals tend to underestimate the level of cognitive

Table 3 Correlation Analysis Results: Association of $\Delta$ temperature with $\Delta \mathrm{PVT}$ and $\Delta$ subjective Outcome Metrics

\begin{tabular}{|c|c|c|c|c|c|c|c|c|c|}
\hline & & \multicolumn{4}{|l|}{ PVT } & \multicolumn{4}{|c|}{ Subjective } \\
\hline & & \multicolumn{2}{|c|}{$\Delta$ Number of Lapses } & \multicolumn{2}{|c|}{$\Delta$ Mean I/RT } & \multicolumn{2}{|c|}{$\Delta$ Sleepiness } & \multicolumn{2}{|c|}{$\Delta$ Fatigue } \\
\hline & & $\mathbf{r}$ & $\mathbf{p}$ & $\mathbf{r}$ & $\mathbf{p}$ & $\mathbf{r}$ & $\mathbf{p}$ & $\mathbf{r}$ & $\mathbf{p}$ \\
\hline \multirow[t]{3}{*}{$\Delta$ Temperature } & $0: 00-2: 00$ & 0.236 & 0.154 & -0.062 & 0.713 & 0.159 & 0.340 & -0.061 & 0.714 \\
\hline & $2: 00-4: 00$ & -0.048 & 0.773 & -0.016 & 0.924 & 0.044 & 0.792 & -0.168 & 0.313 \\
\hline & $4: 00-6: 00$ & $-0.374 *$ & 0.021 & $0.372 *$ & 0.021 & -0.174 & 0.297 & -0.289 & 0.078 \\
\hline
\end{tabular}

Notes: ${ }^{*} p<0.05$. The magnitudes of the relative changes in the adjacent time marked as $\Delta$ subjective $(\Delta$ fatigue and $\Delta$ sleepiness), $\Delta$ objective $(\Delta$ number of lapses and $\Delta$ mean I/RT), and $\Delta$ physiological ( $\Delta$ temperature) outcome metrics. 


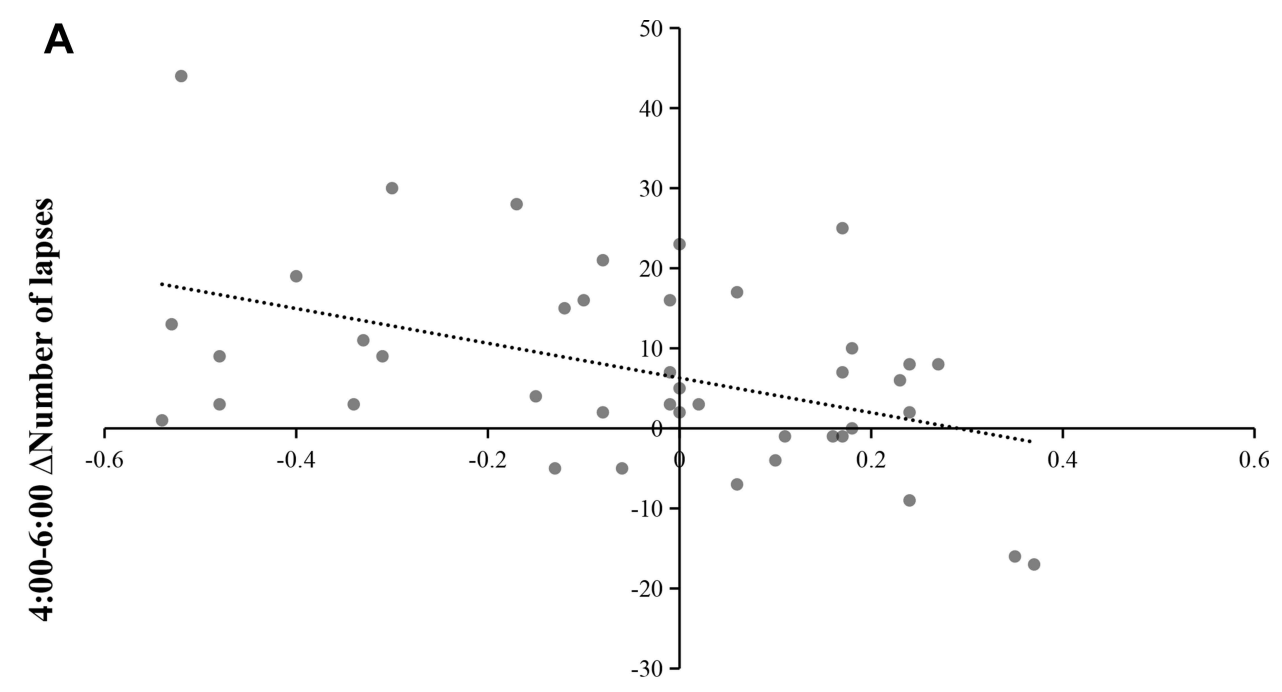

4:00-6:00 $\Delta$ Temperature

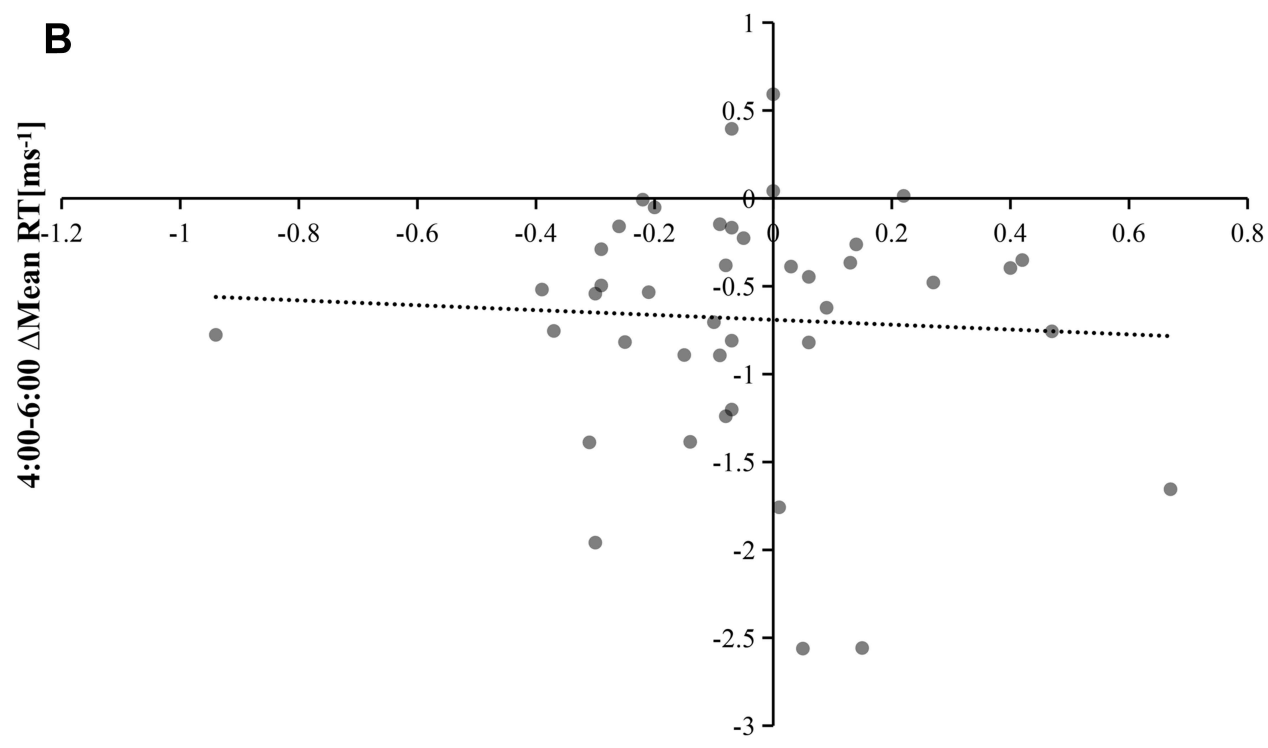

4:00-6:00 $\Delta$ Temperature

Figure 4 The correlations of the changes of subjective ratings/objective performance with the changes of body temperature during the period of prolonged wakefulness. (A) $\Delta$ Number of lapses between 4:00 and 6:00, (B) $\Delta$ Mean I/RT between 4:00 and 6:00.

impairment during the period of extended wakefulness. ${ }^{38}$ The previous study also found the impact of sleep restriction was greater on neurobehavioral performance than that on subjective alertness, especially during the biological night. ${ }^{39}$ There was no relationship between the magnitudes of the subjective and objective alertness impairments during 27 hours of continuous wakefulness. ${ }^{14}$ Similarly, a recent forced desynchrony study revealed the disassociation between subjective alertness and objective performance, and this dissociation may be detrimental to safety during work or daily activities. ${ }^{40}$ Here, we further found that the dissociation of the magnitudes of subjective and objective alertness deteriorations mainly occurs around the minimum of the body temperature rhythm.

Body temperature has been reported to be an underlying mechanism regulating objective performance. Welldocumented studies have indicated that a decreased body temperature is correlated with deteriorated performance and alertness. ${ }^{9,29,31}$ Consistent with previous research, we found that the changes in body temperature were associated with the rate of decrements in objective alertness between 4:00 and 6:00. The more body temperature 
decreased, the more deficits of cognitive performance suffered between 4:00 and 6:00. The previous forced desynchrony studies indicated that neurobehavioral function decreased with the increasing hours awake and was worst during the biological night near the minimum of the body temperature rhythm. ${ }^{41-43}$ Under natural conditions with awake time at 7:00 am and bedtime at 11:00 $\mathrm{pm}$, body temperature falls to reach the trough of $36.5^{\circ} \mathrm{C}$ at 4:00 am. ${ }^{44,45}$ In the present study, objective performance suffered significant decrements when body temperature reaches a minimum between 4:00 and 6:00. The circadian rhythm of body temperature paralleled the circadian rhythm of performance. ${ }^{42}$ The daily cycle in body temperature and behavior performance, such as vigilance, cognition, and sleep-wakefulness cycle, is driven by the circadian pacemaker located in the suprachiasmatic nucleus (SCN) of the hypothalamus. ${ }^{46-48}$ The SCN is essential for maintaining circadian rhythm and synchronizing brain oscillators and physiological status. ${ }^{49,50}$ The circadian rhythm of body temperature has been recognized as a reliable phase marker of the circadian system. ${ }^{51}$ The correlation between objective performance and body temperature can be partly attributable to the regulation of the SCN. Body temperature as a key external indicator of the endogenous circadian rhythm in humans may predict cognitive performance. ${ }^{49}$

Extended wakefulness is a common phenomenon in modern society. Numerous people are used to staying up late at night or working on the night shift. Lack of sleep is a major contributor to work-related and traffic incidents. As observed in this study, subjective and objective alertness showed high vulnerability with an increased duration of wakefulness. It had better not stay up late at night to reduce the chance of accidents. Moreover, individuals may suffer a major deterioration of objective performance but perceive only modest alertness decrements during the period of extended wakefulness. It is dangerous to underestimate the magnitude of cognitive impairment. Finally, it may be a trend to predict the change in cognitive performance by indicators that are easy to measure, such as body temperature.

\section{Limitation and Future Direction}

Several limitations have to be taken into account in the present study. First, participants are age-limited, ranging from 19 to 24 years old. Age is a major factor that may contribute to attentional failures during the period of prolonged wakefulness. ${ }^{52}$ Therefore, the present results may not apply to adolescents or old adults. Second, our results are based on the acute effect of prolonged wakefulness. It is unclear whether the results are valid for the chronic effect of prolonged wakefulness with successive nights. Third, the present study mainly used behavioral and physiological methods. In the future, researchers may consider combining with fMRI, EEG, and the electrodermal activity ${ }^{53,54}$ to explore further the dissociation between objective performance and subjective ratings during prolonged wakefulness time. Fourth, other physiological factors except body temperature may contribute to the changes in objective vigilance, such as cortisol and melatonin during the extended wakeful time. Endogenous melatonin is used alone or combined with core body temperature as a phase marker of the endogenous circadian pacemaker located in the SCN. ${ }^{8}$ Therefore, endogenous melatonin might also be a valuable physiological index that regulates the objective performance during prolonged wakefulness. It is necessary to explore more physiological indexes contributing to the changes in objective vigilance between 4:00 and 6:00 in future research. Fifth, the present study chose the habitual bedtime (0:00 to $6: 00$ am) as the time window of observation to explore the variation tendency of objective and subjective alertness and the dissociation between them. Future researches could consider adding an absolute baseline (eg, 22:00) before habitual sleeping time. Finally, body temperature and sleep-wakefulness cycle are both driven by the circadian pacemaker located in the suprachiasmatic nucleus ( $\mathrm{SCN}$ ) of the hypothalamus. As a result, body temperature might also correlate with vigilance during the daytime. The present study focused on exploring the correlation of body temperature and the objective alertness during the habitual bedtime. Future researches could consider to correlate body temperature with vigilance during the daytime to further uncover the relation between body temperature and behavioral performance.

\section{Conclusion}

The current study found that both subjective and objective alertness deteriorated after prolonged wakefulness of one night, but the magnitudes of decrements in subjective alertness were temporally inconsistent with the performance deficits with the increase of waking time. The significant decrements of objective performance between 4:00 and 6:00 are associated with body temperature rhythm. The current findings revealed a broad range of negative consequences of extended wakefulness, and individuals tend to underestimate their cognitive impairment level during extended wakefulness time. Furthermore, body temperature may be a valuable indicator to predict the change in cognitive performance. 


\section{Funding}

This work was supported by Guangdong Basic and Applied Basic Research Foundation, China (No. 2019A15 15012182) and Natural Science Foundation of SZU (No. 827-000259).

\section{Disclosure}

The authors report no conflicts of financial or academic interest. All authors have read and approved the manuscript.

\section{References}

1. Killgore WD. Effects of sleep deprivation on cognition. Prog Brain Res. 2010;185:105-129.

2. Zhou X, Ferguson SA, Matthews RW, et al. Sleep, wake and phase dependent changes in neurobehavioral function under forced desynchrony. Sleep. 2011;34(7):931-941. doi:10.5665/SLEEP.1130

3. Kaida K, Takahashi M, Akerstedt T, et al. Validation of the Karolinska sleepiness scale against performance and EEG variables. Clin Neurophysiol. 2006;117(7):1574-1581. doi:10.1016/j.clinph.2006.03.011

4. MacLean AW, Fekken GC, Saskin P, Knowles JB. Psychometric evaluation of the Stanford sleepiness scale. J Sleep Res. 1992;1 (1):35-39. doi:10.1111/j.1365-2869.1992.tb00006.x

5. Miley AA, Kecklund G, Akerstedt T. Comparing two versions of the Karolinska Sleepiness Scale (KSS). Sleep Biol Rhythms. 2016;14 (3):257-260. doi:10.1007/s41105-016-0048-8

6. Basner M, Dinges DF. Maximizing sensitivity of the psychomotor vigilance test (PVT) to sleep loss. Sleep. 2011;34(5):581-591. doi:10.1093/sleep/34.5.581

7. Li Y, Vgontzas A, Kritikou I, et al. Psychomotor vigilance test and its association with daytime sleepiness and inflammation in sleep apnea: clinical implications. J Clin Sleep Med. 2017;13(9):1049-1056. doi: $10.5664 /$ jcsm. 6720

8. Cajochen C, Chellappa S, Schmidt C. What keeps us awake?- the role of clocks and hourglasses, light, and melatonin. Int Rev Neurobiol. 2010;93(93):57-90.

9. Carrier J, Monk TH. Circadian rhythms of performance: new trends. Chronobiol Int. 2000;17(6):719-732. doi:10.1081/CBI-100102108

10. Satterfield BC, Wisor JP, Schmidt MA, Van Dongen HPA. Time-ontask effect during sleep deprivation in healthy young adults is modulated by dopamine transporter genotype. Sleep. 2017;40(12). doi:10.1093/sleep/zsx167

11. Veksler BZ, Gunzelmann G. Functional equivalence of sleep loss and time on task effects in sustained attention. Cogn Sci. 2018;42 (2):600-632. doi:10.1111/cogs.12489

12. Cajochen C, Blatter K, Wallach D. Circadian and sleep-wake dependent impact on neurobehavioral function. Psychol Belg. 2004;44(12):59-80. doi:10.5334/pb.1017

13. Chua EC, Tan WQ, Yeo SC, et al. Heart rate variability can be used to estimate sleepiness-related decrements in psychomotor vigilance during total sleep deprivation. Sleep. 2012;35(3):325-334. doi:10. 5665/sleep. 1688

14. Leproult R, Colecchia EF, Berardi AM, Stickgold R, Kosslyn SM, Van Cauter E. Individual differences in subjective and objective alertness during sleep deprivation are stable and unrelated. $A m$ J Physiol Regul Integr Comp Physiol. 2003;284(2):R280-R290. doi:10.1152/ajpregu.00197.2002

15. Paech GM, Banks S, Pajcin M, et al. Caffeine administration at night during extended wakefulness effectively mitigates performance impairment but not subjective assessments of fatigue and sleepiness. Pharmacol Biochem Behav. 2016;145:27-32. doi:10.10 16/j.pbb.2016.03.011
16. Kanayama N, Sato A, Ohira H. Crossmodal effect with rubber hand illusion and gamma-band activity. Psychophysiology. 2007;44 (3):392-402. doi:10.1111/j.1469-8986.2007.00511.x

17. Posada-Quintero HF, Reljin N, Bolkhovsky JB, Orjuela-Canon AD, Chon KH. Brain activity correlates with cognitive performance deterioration during sleep deprivation. Front Neurosci. 2019;13:1001. doi:10.3389/fnins.2019.01001

18. Johnson BK. Physiology of the autonomic nervous system. In: Farag E, Argalious M, Tetzlaff J, Sharma D, editors. Basic Sciences in Anesthesia. Cham: Springer; 2018:355-364.

19. Hibi M, Kubota C, Mizuno T, et al. Effect of shortened sleep on energy expenditure, core body temperature, and appetite: a human randomised crossover trial. Sci Rep. 2017;7(1):39640. doi:10.1038/ srep39640

20. Szymusiak R. Body temperature and sleep. Handb Clin Neurol. 2018;156:341-351.

21. Te Lindert BHW, Van Someren EJW. Skin temperature, sleep, and vigilance. Handb Clin Neurol. 2018;156:353-365.

22. Vaara J, Kyrolainen H, Koivu M, Tulppo M, Finni T. The effect of 60-h sleep deprivation on cardiovascular regulation and body temperature. Eur J Appl Physiol. 2009;105(3):439-444. doi:10.10 07/s00421-008-0921-5

23. Schwartz WJ, Klerman EB. Circadian neurobiology and the physiologic regulation of sleep and wakefulness. Neurol Clin. 2019;37 (3):475-486. doi:10.1016/j.ncl.2019.03.001

24. Zhong X, Hilton HJ, Gates GJ, et al. Increased sympathetic and decreased parasympathetic cardiovascular modulation in normal humans with acute sleep deprivation. J Appl Physiol. 2005;98 (6):2024-2032. doi:10.1152/japplphysiol.00620.2004

25. Meier-Ewert HK, Ridker PM, Rifai N, et al. Effect of sleep loss on C-reactive protein, an inflammatory marker of cardiovascular risk. $J$ Am Coll Cardiol. 2004;43(4):678-683. doi:10.1016/j.jacc.2003.07.050

26. Yang H, Haack M, Gautam S, Meier-Ewert HK, Mullington JM. Repetitive exposure to shortened sleep leads to blunted sleep-associated blood pressure dipping. J Hypertens. 2017;35 (6):1187-1194. doi:10.1097/HJH.0000000000001284

27. Zoccoli G, Amici R. Sleep and autonomic nervous system. Curr Opion Physiol. 2020;15:128-133. doi:10.1016/j. cophys.2020.01.002

28. Goel N. Neurobehavioral effects and biomarkers of sleep loss in healthy adults. Curr Neurol Neurosci Rep. 2017;17(11):89. doi:10. 1007/s11910-017-0799-x

29. Wright KP, Hull JT, Czeisler CA. Relationship between alertness, performance, and body temperature in humans. Am J Physiol Regul Integr Comp Physiol. 2002;283(6):R1370-1377. doi:10.1152/ ajpregu.00205.2002

30. Molzof HE, Prapanjaroensin A, Patel VH, Mokashi MV, Gamble KL, Patrician PA. Misaligned core body temperature rhythms impact cognitive performance of hospital shift work nurses. Neurobiol Learn Mem. 2019;160:151-159. doi:10.1016/j. nlm.2019.01.002

31. Valdez P, Ramírez C, García A. Circadian rhythms in cognitive performance: implications for neuropsychological assessment. Chrono Physiol Ther. 2012;2:81-92. doi:10.2147/CPT.S32586

32. Stevens JP. Outliers and influential data points in regression analysis. Psychol Bull. 1984;95(2):334. doi:10.1037/0033-2909.95.2.334

33. Doran SM, Van Dongen HP, Dinges DF. Sustained attention performance during sleep deprivation: evidence of state instability. Arch Ital Biol. 2001;139(3):253-267.

34. Shenfield L, Beanland V, Filtness A, Apthorp D. The impact of sleep loss on sustained and transient attention: an EEG study. PeerJ. 2020;8:e8960. doi:10.7717/peerj.8960

35. Dinges DF, Powell JW. Microcomputer analyses of performance on a portable, simple visual RT task during sustained operations. Behav Res Methods Instrum Comput. 1985;17(6):652-655. doi:10.3758/ BF03200977 
36. Basner M, Mollicone D, Dinges DF. Validity and sensitivity of a brief psychomotor vigilance test (PVT-B) to total and partial sleep deprivation. Acta Astronaut. 2011;69(11-12):949-959. doi:10.1016/ j.actaastro.2011.07.015

37. Dijk DJ, von Schantz M. Timing and consolidation of human sleep, wakefulness, and performance by a symphony of oscillators. $\mathrm{J}$ Biol Rhythms. 2005;20(4):279-290. doi:10.1177/0748730405278292

38. Dinges DF, Pack F, Williams K, et al. Cumulative sleepiness, mood disturbance, and psychomotor vigilance performance decrements during a week of sleep restricted to 4-5 hours per night. Sleep. 1997;20 (4):267-277

39. Zhou X, Ferguson SA, Matthews RW, et al. Mismatch between subjective alertness and objective performance under sleep restriction is greatest during the biological night. J Sleep Res. 2012;21(1):40-49. doi:10.1111/j.1365-2869.2011.00924.x

40. McHill AW, Hull JT, Wang W, Czeisler CA, Klerman EB. Chronic sleep curtailment, even without extended (>16-h) wakefulness, degrades human vigilance performance. Proc Natl Acad Sci U S A. 2018;115(23):6070-6075. doi:10.1073/pnas.1706694115

41. Czeisler CA, Dijk D-J, Duffy JF. Entrained phase of the circadian pacemaker serves to stabilize alertness and performance throughout the habitual waking day; 1994.

42. Dijk DJ, Duffy JF, Czeisler CA. Circadian and sleep/wake dependent aspects of subjective alertness and cognitive performance. J Sleep Res. 1992;1(2):112-117. doi:10.1111/j.1365-2869.1992.tb00021.x

43. Wyatt JK, Ritz-de Cecco A, Czeisler CA, Dijk DJ. Circadian temperature and melatonin rhythms, sleep, and neurobehavioral function in humans living on a 20-h day. Am J Physiol. 1999;277(4 Pt 2): R1152-1163. doi:10.1152/ajpregu.1999.277.4.r1152

44. Refinetti R. The circadian rhythm of body temperature. Front Biosci. 2010;15(3):564-594. doi:10.2741/3634

45. Refinetti R. Circadian rhythmicity of body temperature and metabolism. Temperature (Austin). 2020;7(4):321-362. doi:10.1080/ 23328940.2020.1743605
46. Deboer T, Detari L, Meijer JH. Long term effects of sleep deprivation on the mammalian circadian pacemaker. Sleep. 2007;30(3):257-262. doi:10.1093/sleep/30.3.257

47. Gent TC, Bassetti C, Adamantidis AR. Sleep-wake control and the thalamus. Curr Opin Neurobiol. 2018;52:188-197. doi:10.1016/j. conb.2018.08.002

48. Saper CB, Scammell TE, Lu J. Hypothalamic regulation of sleep and circadian rhythms. Nature. 2005;437(7063):1257-1263. doi:10.1038/ nature 04284

49. Moore-Ede MC, Sulzman FM, Sulzman FM, Fuller CA. The Clocks That Time Us: Physiology of the Circadian Timing System. Harvard University Press; 1982.

50. Zhao ZD, Yang WZ, Gao C, et al. A hypothalamic circuit that controls body temperature. Proc Natl Acad Sci U S A. 2017;114 (8):2042-2047. doi:10.1073/pnas.1616255114

51. Gradisar M, Lack L. Relationships between the circadian rhythms of finger temperature, core temperature, sleep latency, and subjective sleepiness. J Biol Rhythms. 2004;19(2):157-163. doi:10.1177/ 0748730403261560

52. Blatter K, Graw P, Munch M, Knoblauch V, Wirz-Justice A, Cajochen C. Gender and age differences in psychomotor vigilance performance under differential sleep pressure conditions. Behav Brain Res. 2006;168(2):312-317. doi:10.1016/j.bbr.2005.11.018

53. Posada-Quintero HF, Bolkhovsky JB, Reljin N, Chon KH. Sleep deprivation in young and healthy subjects is more sensitively identified by higher frequencies of electrodermal activity than by skin conductance level evaluated in the time domain. Front Physiol. 2017;8:409. doi:10.3389/fphys.2017.00409

54. Romine W, Banerjee T, Goodman G. Toward sensor-based sleep monitoring with electrodermal activity measures. Sensors (Basel). 2019;19(6):1417. doi:10.3390/s19061417
Nature and Science of Sleep

\section{Publish your work in this journal}

Nature and Science of Sleep is an international, peer-reviewed, open access journal covering all aspects of sleep science and sleep medicine, including the neurophysiology and functions of sleep, the genetics of sleep, sleep and society, biological rhythms, dreaming, sleep disorders and therapy, and strategies to optimize healthy sleep.
The manuscript management system is completely online and includes a very quick and fair peer-review system, which is all easy to use. Visit http://www.dovepress.com/testimonials.php to read real quotes from published authors. 